\title{
Imaging of surface-breaking concrete cracks using transient elastic waves
}

\author{
Pei-Ling Liu, Chong-Dao Tsai and Tsung-Tsong Wu \\ Institute of Applied Mechanics, National Taiwan University, Taipei, Taiwan
}

\begin{abstract}
This study develops an imaging method to determine the depth and inclination of a surface-breaking crack in a concrete structure. The migration method in reflection seismology is adopted to process the surface response of the concrete structure recorded in a transient elastic wave test. An image is constructed that shows the location of the crack tip. The response curves are processed further by the quadratic interpolation method to determine the location of the crack tip. Then, a solid line is superposed on the image to represent the crack. Numerical examples are presented to illustrate the effectiveness of the proposed method to display cracks of various dip angles and lengths. Two model tests verify that this method can detect real cracks successfully. Copyright (C) 1996 Elsevier Science Ltd.
\end{abstract}

Keywords: concrete, surface-breaking crack, transient waves

Recently, the transient elastic wave test has been adopted by many researchers in the nondestructive examination of concrete structures. Lin and Sansalone ${ }^{[1]}$ have used the impact echo method to detect flaws in concrete beams and columns. Wu et al. ${ }^{[2]}$ have utilized the transient elastic wave to measure the elastic constants of a concrete specimen. Wu et al.$^{[3]}$ have also used the phase information to detect the depth of a vertical crack in concrete structures. The same problem has been addressed by $\mathrm{Lin}$ and $\mathrm{Su}^{[4]}$ using the impact echo method.

In the elastic wave test of concrete structures, a source is applied on the surface of the concrete to generate waves in the structure. If the waves encounter an interface or inhomogeneity, they will be reflected, refracted or diffracted. Then, the surface response of the concrete is measured and recorded for data processing.

The elastic wave test has its parallel in seismic exploration. In the seismic test, a source is also applied on the surface of the earth. Then, the surface response of the earth is received by an array of geophones. Finally, the signals are processed to produce an image of the geological structure. Since the two tests are both based on elastic wave propagation, it is expected that the imaging method in seismology can also be applied to process concrete signals.

The procedure of signal processing in reflection seismology mainly includes the following steps: static and dynamic corrections, horizontal stacking, migration, and velocity analysis ${ }^{[5]}$. Because there are fundamental differences between a cracked concrete structure and the earth's structure, most of these steps are either unsuitable or unnecessary in crack detection except migration. For example, the earth usually has a layered structure and the dip angles of the layers are usually small. A concrete block, on the other hand, is homogeneous from a macroscopic viewpoint. There are no layer interfaces in the concrete, but cracks may exist, and the cracks usually have high dip angles.

Migration can compress diffraction signals back to their originating point and depict the location of the diffraction point. The tip of a crack is certainly a diffraction point. Therefore, the idea of migration is adopted in this paper to locate the tip of a surfacebreaking crack in concrete.

\section{Migration}

Consider an elastic half-plane with a surface-breaking crack. When a source is applied on the free boundary, elastic waves are generated and propagated in the medium. Waves reaching the crack will be reflected, and the waves reaching the crack tip will be diffracted and arrive at the other side of the crack. In addition, surface waves will be generated. 
The amplitude of the surface wave is much larger than that of the body wave. If the source and receiver are on the same side of the crack opening, the surface response is dominated by the surface wave. Unfortunately, surface waves do not contain any useful information about the crack. Therefore, the source and receiver should be placed on the opposite sides of the crack opening. In that case, the signals received are mostly diffracted waves because the surface waves are blocked by the crack. Therefore, it is difficult to construct a complete image of the crack using such signals. However, one may migrate the diffracted signals to locate the crack tip.

The principles of migration are as follows: consider a signal that is emitted from the source, diffracted at a point, and arrives at the receiver. Suppose the travel time of the diffracted signal is $t$, and the wave velocity is $v$. Then, the travel distance of the signal is $v t$. If the diffraction path is unknown, any point in the medium with the same travel distance is a possible diffraction point. Therefore, the diffraction point should fall on an ellipse with the source and receiver as its foci, as shown in Figure 1. If two ellipses are drawn using different diffracted signals, the diffracted point must be located at the intersection of the two ellipses.

A migration image can be constructed following this idea. We firstly draw a blank half-plane. Then, an ellipse is constructed for each of the data points on the response curve according to the travel distance. Since the amplitude of the data points varies, the elliptic trajectories are of different magnitudes. Representing the magnitude by greyscale, we get a greyscale image. Since there is a visible change in the response curve when the first diffracted signal arrives, the ellipse of the first diffraction can be located on the image. If more than one response curve is migrated, the crack tip is simply located at the common intersection of the ellipses of the first arrivals.

The actual procedure for constructing a migration image is as follows

1. Divide the imaging zone into $n_{x} \times n_{z}$ pixels.

2. For pixel $(i, j)$, compute its distance to the source, $d_{s}$, and to the receiver, $d_{\mathrm{r}}$. Then, calculate the time it takes for a signal to travel from the source, passing the pixel, to the receiver:

$$
t_{i j}=\frac{d_{\mathrm{r}}+d_{\mathrm{s}}}{v}
$$

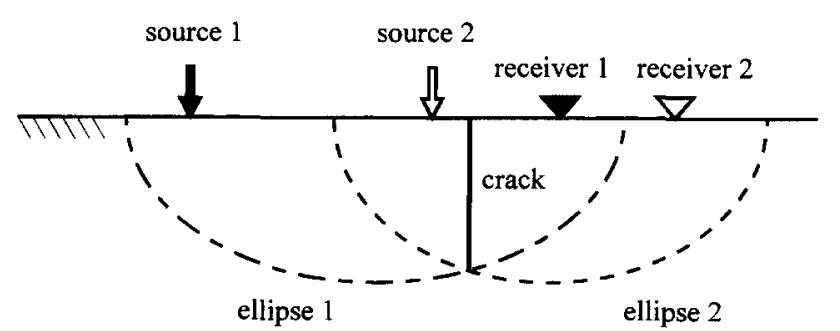

Figure 1 Principles of migration where $t_{i j}$ is the travel time, and $v$ is the velocity of the $P$ wave.

3. Sum up the amplitude of all the response curves at time $t_{i j}$ :

$$
A(i, j)=\sum_{n=1}^{N} r_{n}\left(t_{i j}\right)
$$

where $N$ is the total number of response curves, and $r_{n}$ is the $n$th curve.

4. Select an upper bound $U$. Then, compute the greyscale for each pixel:

$$
G(i, j)=\left\{\begin{array}{cl}
-1 & A(i, j) \leq-U \\
\frac{A(i, j)}{U} & -U \leq A(i, j) \leq U \\
1 & A(i, j) \geq U
\end{array}\right.
$$

When $G(i, j)=1$, the pixel is white; when $G(i, j)=-1$, the pixel is black.

\section{Construct a greyscale image according to $G(i, j)$.}

The image is constructed using the modulated amplitude $G(i, j)$ because the response curve varies slowly when the first diffraction arrives. If $A(i, j)$ is used to construct the image, the greyscale around the first-arrival ellipse changes gradually. As a result, the first-arrival ellipses do not show clearly, neither does their intersection. The purpose of modulating the amplitude is to exaggerate the variation of the waveform so that the intersection can be easily identified. Apparently, as the upper bound $U$ decreases, the ellipse of the first arrival becomes more and more clear.

\section{Crack imaging}

The greyscale image obtained by migration can only depict the location of the crack tip, not the crack itself. To make the image more easily read, a line segment is superposed on the greyscale image to represent the crack.

The crack tip is simply located at the intersection of the first-arrival ellipses. Therefore, to draw the line segment, the travel time of the first arrivals is determined first. Then, simultaneous equations are solved to find the intersection of the ellipses. Once the crack tip is found, we simply draw a line connecting the crack opening and crack tip.

The simplest way to determine the travel time of the first arrival, denoted $t_{0}$, is to select a threshold $T$. Then, the time the curve amplitude exceeds $T$ is an approximation of the arrival time. This value is an overestimate of $t_{0}$. Its accuracy depends on how fast the curve ascends or descends when the signal arrives.

In order to improve the results, quadratic interpolation is adopted here to get a better estimate of the arrival time. A quadratic curve is used to interpolate the data points around the arrival time. Then, the root of the quadratic 
curve is the new approximation of $t_{0}$. The interpolation is repeated several times until convergence is achieved.

There are two parameters to be selected in this approach: the threshold $T$ and the interpolation range $D$. Theoretically, $T$ should be as small as possible so that one gets a good approximation of $t_{0}$ in the first step. However, if $T$ is too small, the process may be triggered by noise. Therefore, $T$ should be slightly larger than the noise level.

The value of $D$ should reflect the waveform of the first arrival, which is mainly influenced by the duration of source and the geometry. Since the geometry of the crack is unknown in real applications, only the duration of the source is considered in this study. Figure 2 shows the influence of source duration on the displacement curve when the first signal arrives. It is seen that as the source duration decreases, the waveform changes more rapidly. Therefore, the value of $D$ should be small when the source duration is short, and vice versa. Experience shows that $20 \%$ of the source duration is a good choice of $D$.

\section{Experimental layout}

It is mentioned that the crack tip is located at the intersection of the first-arrival ellipses. If the positions of the source and receiver remain unchanged in the tests, the first-arrival ellipses coincide with each other, and no intersection can be found. The intersection becomes more and more clear as the ellipses become apart. Therefore, the layout of the source and receiver should be designed carefully in the tests.

Consider the crack in Figure 3. The surface opening and the tip of the crack are located at $(0,0)$ and $(c, d)$, respectively. Suppose the locations of the source and receiver are respectively $\left(s_{1}, 0\right)$ and $\left(r_{1}, 0\right)$ in the first test, and $\left(s_{2}, 0\right)$ and $\left(r_{2}, 0\right)$ ir the second test. Then, the two first-arrival ellipses satisfy:

$$
\left(\frac{x-\frac{r_{i}+s_{i}}{2}}{a_{i}}\right)^{2}+\left(\frac{z}{b_{i}}\right)^{2}=1 \quad i=1,2
$$

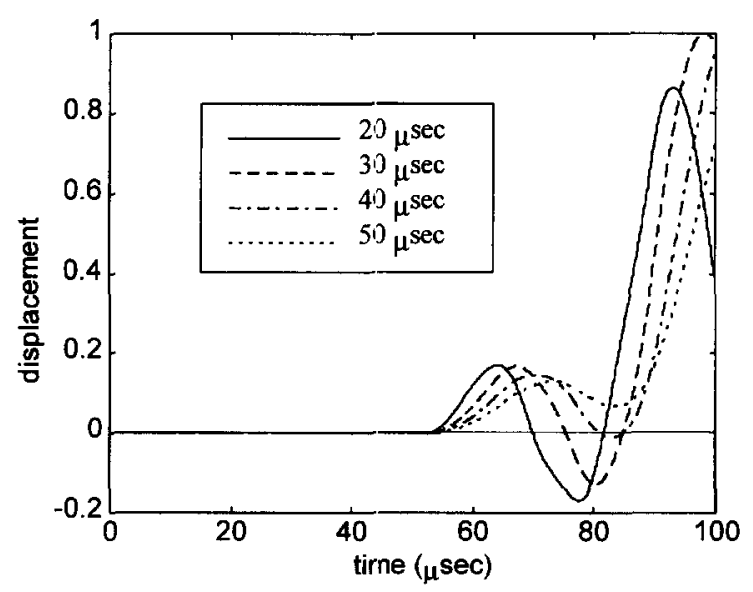

Figure 2 Influence of source duration on the displacement curve.

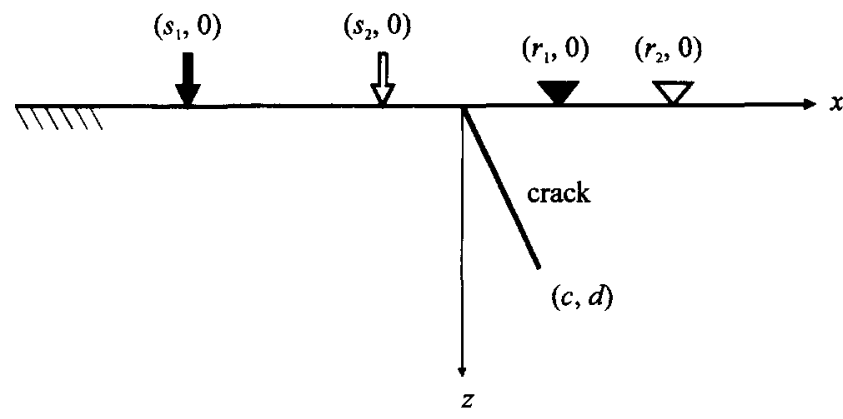

Figure 3 Test layout

where:

$$
\begin{aligned}
& a_{i}=\frac{1}{2}\left(\sqrt{\left(s_{i}-c\right)^{2}+d^{2}}+\sqrt{\left(r_{i}-c\right)^{2}+d^{2}}\right) \\
& b_{i}=\sqrt{a_{i}^{2}-\left(\frac{r_{i}-s_{i}}{2}\right)^{2}}
\end{aligned}
$$

The slopes of the ellipses at the intersection are:

$$
\left.\frac{d z}{\mathrm{~d} x}\right|_{i}(c, d)=\left[\frac{\left(r_{i}-s_{i}\right)^{2}}{4 a_{i}^{2}}-1\right]\left(\frac{c}{d}-\frac{r_{i}+s_{i}}{2 d}\right)
$$

The surface plot in Figure 4 shows the influence of $s_{i}$ and $r_{i}$ on $\mathrm{d} z / \mathrm{d} x$. The two axes $S=(s-c) / d$ and $R=(r-c) / d$ in the figure are dimensionless locations. When $S+R>0$, the slope is positive, and $|\mathrm{d} z / \mathrm{d} x|$ increases as $S$ and $R$ increase. When $S+R<0$, the slope is negative, and $|\mathrm{d} z / \mathrm{d} x|$ increase as $S$ and $R$ decrease.

As the difference of the two slopes increases, the image of the crack tip becomes sharp. Therefore, it is advisable to arrange the source and receiver such that $S+R \gg 0$ in the first test and $S+R \ll 0$ in the second test. However, there are limitations on $r_{i}$ and $s_{i}$. Since the source and receiver must be located on the opposite sides of the crack opening, $r_{i}$ and $s_{i}$ cannot be made large or small simultaneously. The best layout one can have is to let $s_{i}=-\varepsilon_{1}<0, r_{1}=l_{1}>0$ and $s_{2}=\varepsilon_{2}>0, r_{2}=-l_{2}<0$, where $\varepsilon_{i}$ are small and $l_{i}$ are large. The key point is to make the two ellipses as separated as possible.

Since exchanging the positions of the source and receiver does not alter the travel time, one can exchange the positions of the source and receiver in the aforementioned layouts. It should be noted that the distance between the source and receiver cannot be too far, otherwise the signal will be too weak and the image will blur.

\section{Numerical examples}

Consider a concrete half-plane with a surface-breaking crack. The longitudinal wave velocity is $4160 \mathrm{~m} / \mathrm{s}$, the transverse wave velocity is $2450 \mathrm{~m} / \mathrm{s}$, and the mass density is $2320 \mathrm{~kg} / \mathrm{m}^{3}$. Suppose two tests are performed on the specimen. In each test, a vertical force is applied on the surface of the concrete, the source time function being a half-sine function with a peak amplitude of $100 \mathrm{~N}$ and a duration of about $20 \mu \mathrm{s}$. 


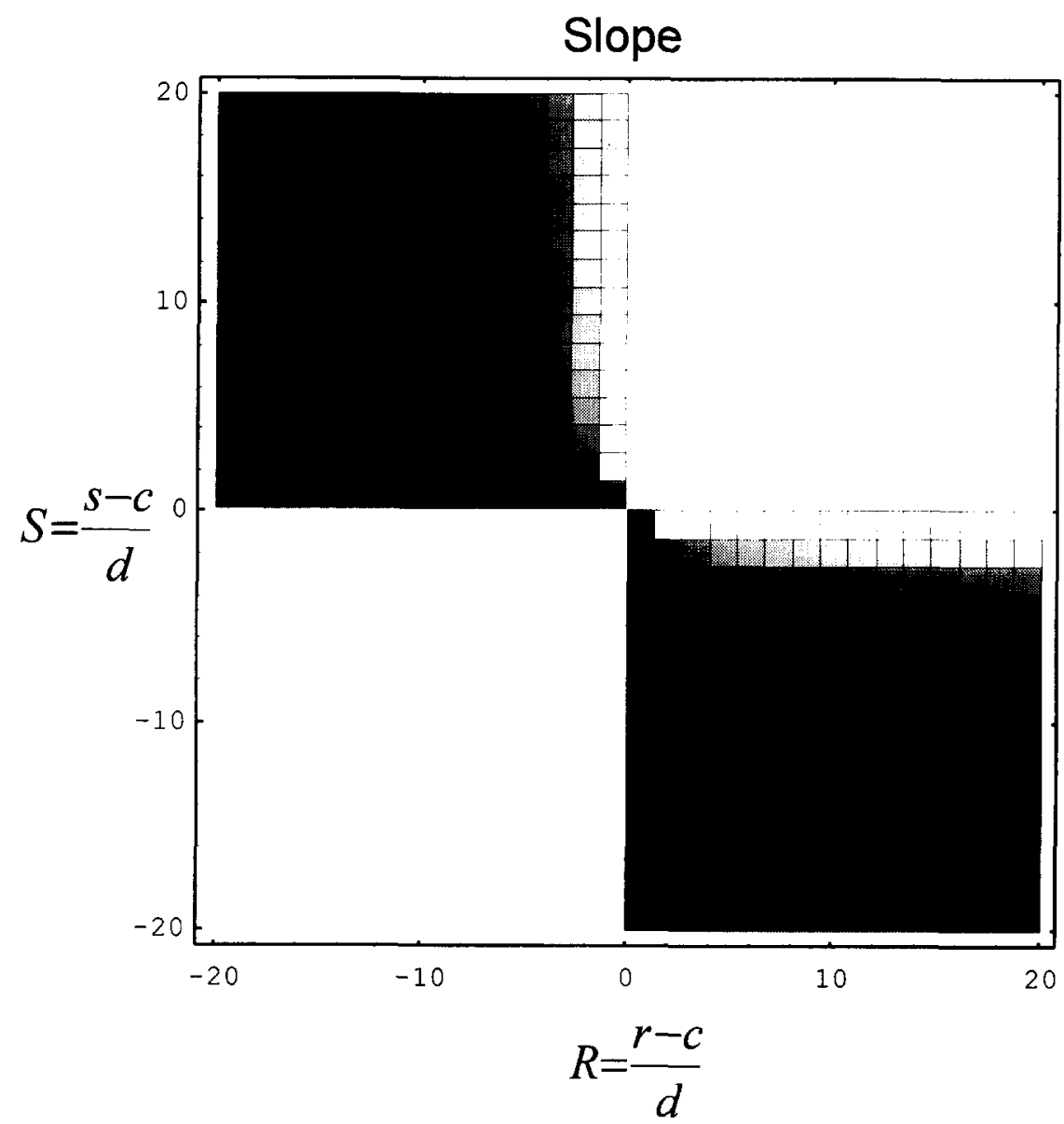

Figure 4 Influence of source and receiver locations on the slope of the first-arrival ellipse at the crack tip

The finite-difference method proposed by Virieux ${ }^{[6]}$ was adopted to compute the response of the specimen. $\mathrm{Wu}$ and Gong ${ }^{[7]}$ have shown that the wave field predicted by this algorithm agrees well with experimental results. The grid intervals were $\Delta x=\Delta z=1 \mathrm{~mm}$ in the simulation, and the time step was $\Delta t=0.1 \mu \mathrm{s}$. These parameters were selected based on the accuracy and stability criteria $^{[6]}$.

First of all, consider a vertical crack, the depth of which is $60 \mathrm{~mm}$. Suppose the test layouts are $s_{1}=-10 \mathrm{~mm}$, $r_{1}=100 \mathrm{~mm}$ and $s_{2}=10 \mathrm{~mm}, r_{2}=-100 \mathrm{~mm}$. The displacement and velocity curves for this test layout are shown in Figure 5. Because of symmetry, the displacement and velocity curves of the two tests are identical.

Figure 6 shows the images obtained by processing the displacement and velocity signals. The symbol $x$ in the image denotes the actual location of the crack tip. In the velocity image, the crack is surrounded by a zerogreyscale zone, corresponding to the quiescent part of the curve before the first signal arrives. Outside the zerogreyscale zone, there is a dark band, corresponding to the valley of the curve next to the first arrival. Outside the dark band is a bright zone, corresponding to the positive signals after the valley. The displacement image is similar to the velocity image. However, since the displacement curve rises directly as the first diffraction comes, there is no dark band around the zero-greyscale zone. Whether in the displacement image or velocity image, the crack tip is located at the V-shaped corner of the zero-greyscale zone.

It is seen that both the displacement and velocity images can depict the location of the crack tip. Moreover, the velocity image is superior to the displacement image. This is because the velocity curve has a more obvious change than the displacement curve as the first signal arrives, as shown in Figure 5.

The crack tip determined by interpolation is rather close to the actual tip location. The crack length obtained is $66.2 \mathrm{~mm}$, which is $10.3 \%$ longer than the actual length. The main reason can be found in Figure 5. It is seen that the response curves do not ascend or descend immediately after the first arrival. There is a time lag of a few $\mu$ s. Fortunately, the estimation is on the safe side from an engineering point of view.

It is mentioned that the upper bound $U$ in Eq. (3) controls the clearness of the image. Figure 7 shows that as $U$ is increased by ten times and a hundred times, the corner of the zero-greyscale zone becomes less and less pointed. Obviously, it is difficult to spot the crack tip by reading such images. Therefore, $U$ should be set as small as possible as long as it exceeds the noise level.

Now, consider the case of a short crack, the length of which is $30 \mathrm{~mm}$. The source and receiver layouts in the 


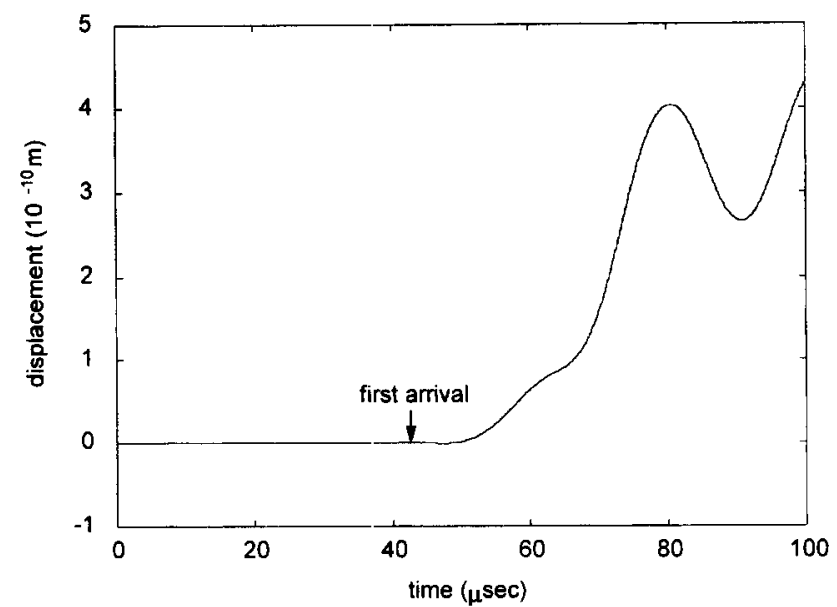

(a)

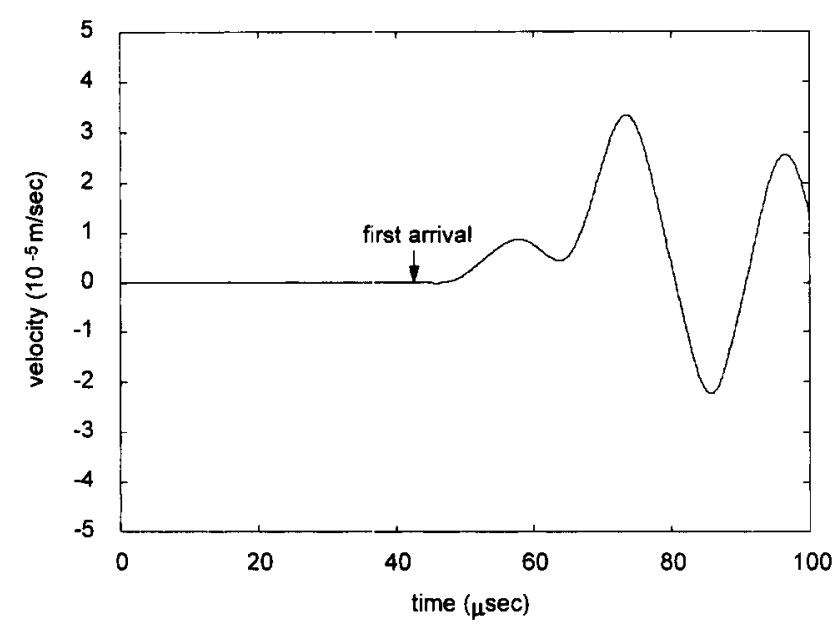

(b)

Figure 5 (a) Displacement and (b) velocity curve of the $60 \mathrm{~mm}$ vertical crack

two tests remain the same. Since the crack length is reduced to a half, the difference between the slopes of the two first-arrival ellipses decreases. Therefore, the corner of the zero-greyscale zone is not as clear as in the first case, as shown in Figure 8 . In that case, one may want to repeat the tests to attain a better image of the crack.

Next, consider a crack with a $45^{\circ}$ dip angle. Such cracks are very common in concrete structures. Suppose the crack tip is at $(60 \mathrm{~mm}, 60 \mathrm{~mm})$, and its length is $60 \sqrt{2} \mathrm{~mm}$. Since the crack orientation is supposedly unknown, the same symmetric test layouts are adopted in this case.

Figure 9 shows the velocity image of the crack. It is seen that outside the zero-greyscale zone, there is a dark region to the left of the crack tip and a bright region to the right of the crack tip. The image shows the location of the crack tip clearly.

The coordinates of the crack tip obtained by interpolation are $(66.7 \mathrm{~mm}, 61.1 \mathrm{~mm})$, the crack length is $90.5 \mathrm{~mm}$, and the dip angle is $42.5^{\circ}$. Hence, the errors of the crack length and dip angle are $6.6 \%$ and $5.5 \%$, respectively, which should be acceptable.

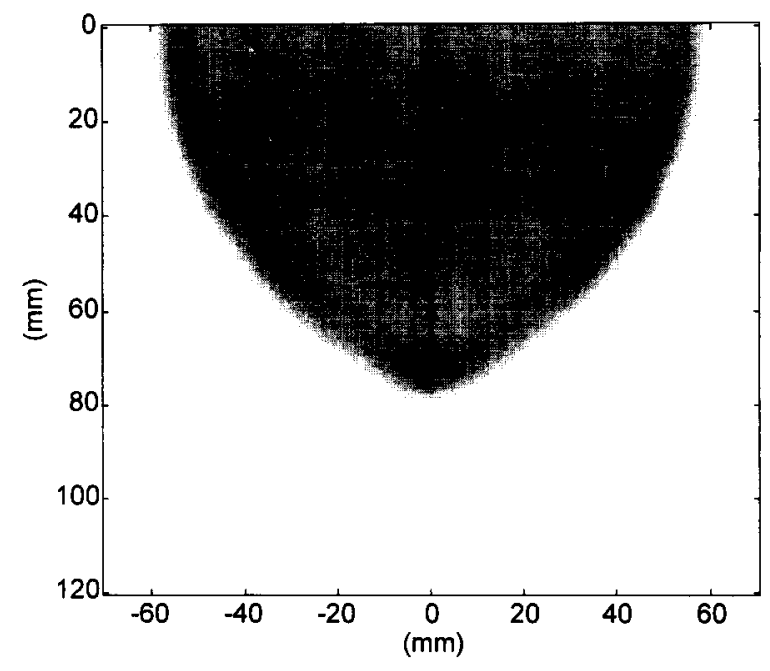

(a)

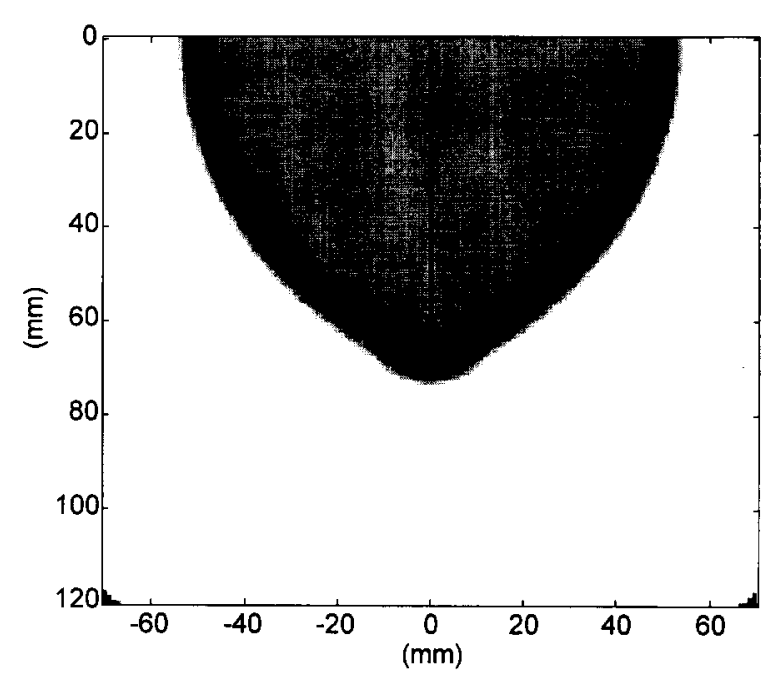

(b)

Figure 6 (a) Displacement and (b) velocity images of the $60 \mathrm{~mm}$ vertical crack

Finally, consider another test layout, which is symmetric with respect to the crack tip, not the crack opening. Let $s_{1}=-10 \mathrm{~mm}$ and $r_{1}=190 \mathrm{~mm} ; s_{2}=130 \mathrm{~mm}$ and $r_{2}=-70 \mathrm{~mm}$. The results of velocity imaging are shown in Figure 10. Apparently, the image is inferior to that in Figure 9. This is because the angle between the tangents of the two first-arrival ellipses is $43^{\circ}$ using the first layout, but only $16^{\circ}$ using the second layout. This example shows the importance of selecting an appropriate test layout.

\section{Model test}

The ability of the proposed method to display a real, three-dimensional crack is examined by two model tests. The experiments were conducted on two concrete specimens. The water-to-cement ratio of the concrete is 0.58 . The maximum size of the coarse aggregate is $1.92 \mathrm{~cm}$, and the cement to fine aggregate and to coarse 


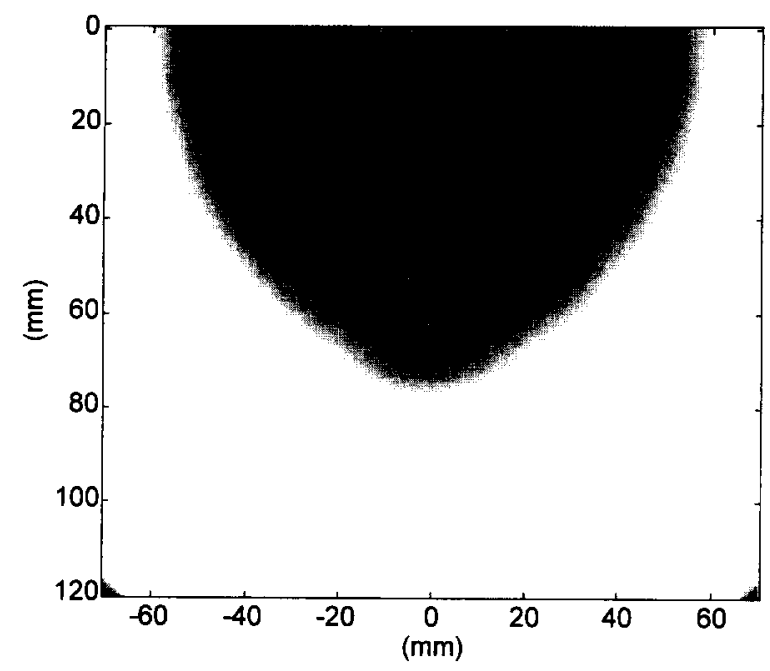

(a)

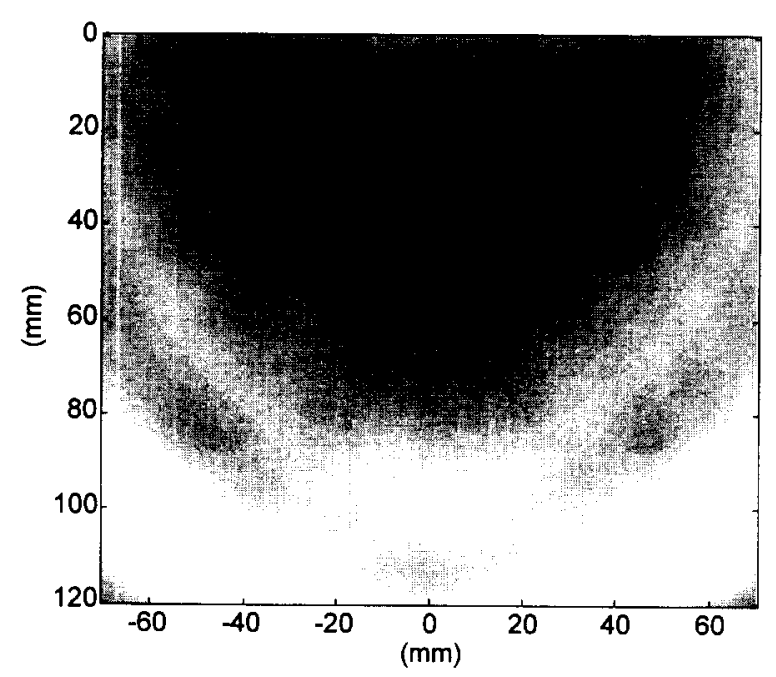

(b)

Figure 7 Velocity images of the $60 \mathrm{~mm}$ vertical crack with $U$ increased by (a) 10 times and (b) 100 times

aggregate ratio is $1: 1.5: 2.5$. The longitudinal and transverse wave velocities and the mass density are the same as in the numerical example. The first specimen has a vertical slit, the width and depth of which are $2.5 \mathrm{~mm}$ and $90 \mathrm{~mm}$, respectively. The second specimen has a slit with $45^{\circ}$ dip angle, the width and length of which are $1.5 \mathrm{~mm}$ and $120 \mathrm{~mm}$, respectively.

A vertical point source was generated by dropping a small steel ball $(4.75 \mathrm{~mm}$ in diameter) on the surface of the specimens. Since the source duration is about $20 \mu \mathrm{s}$, the dominant frequency is about $50 \mathrm{kHz}$. The wavelength of the induced longitudinal wave is greater than $80 \mathrm{~mm}$, which is much larger than the slit widths and the maximum size of the aggregate. Therefore, the slit can be considered as a crack, and the specimen can be considered homogeneous.

In the experiments, an NBS conical transducer was used to measure the vertical displacement signals on the surface of the specimens. The received voltage signals were then fitted in a preamplifier and recorded by a

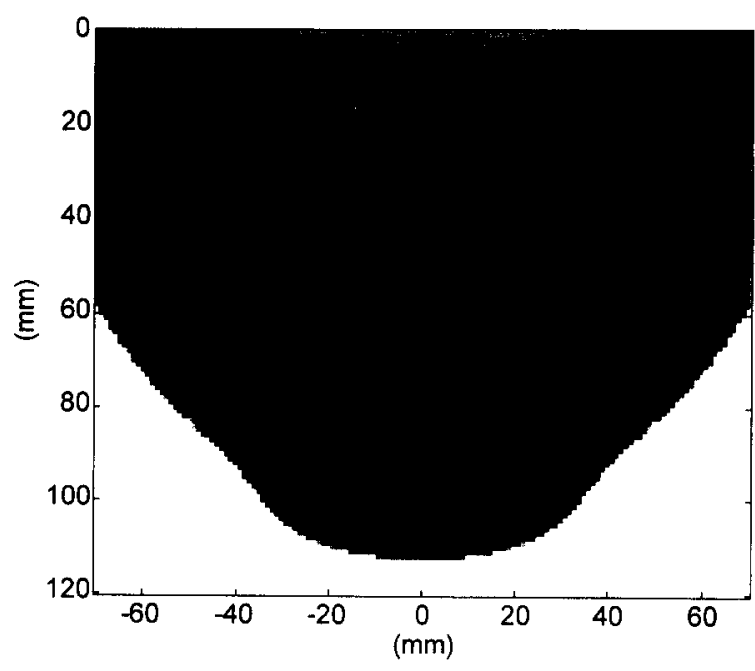

(a)

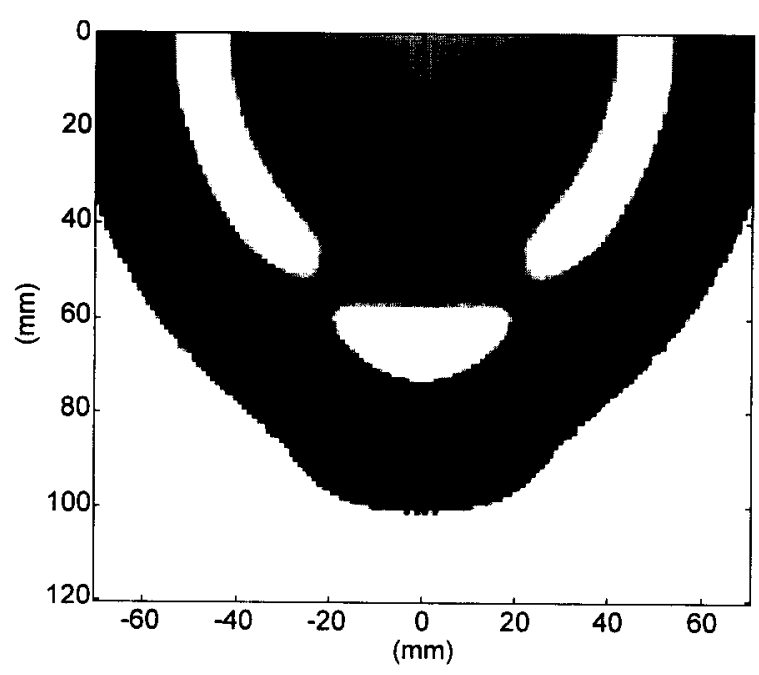

(b)

Figure 8 (a) Displacement and (b) velocity images of the $30 \mathrm{~mm}$ vertical crack

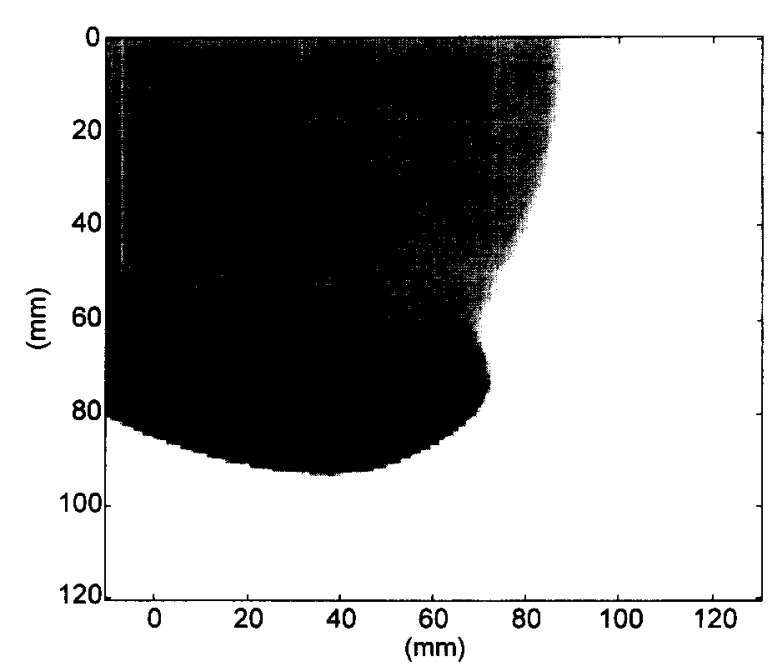

Figure 9 Velocity image of the $45^{\circ}$ crack; $\left(s_{1}, r_{1}\right)=(-10,100)$, $\left(s_{2}, r_{2}\right)=(10,-100)$ 


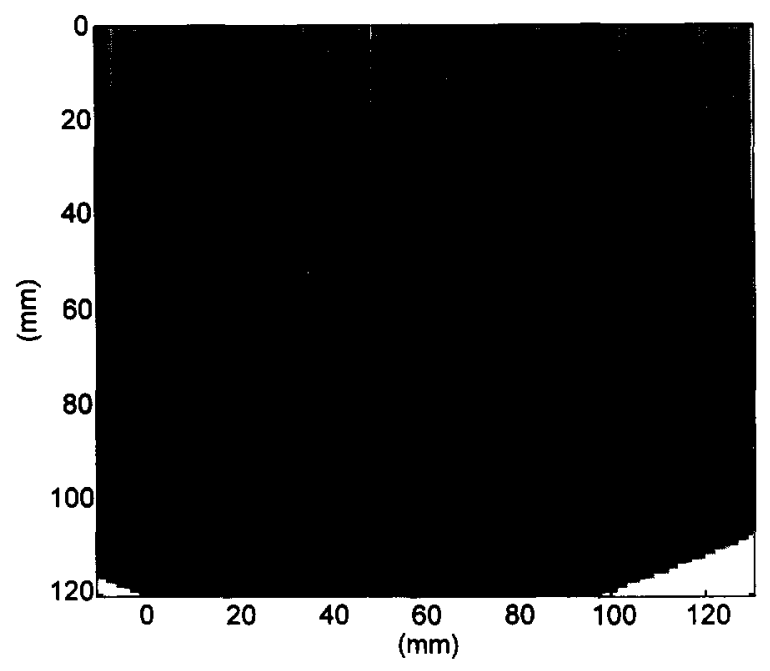

Figure 10 Velocity image of the $45^{\circ}$ crack; $\left(s_{1}, r_{1}\right)=(-10$, $190),\left(s_{2}, r_{2}\right)=(130,-70)$

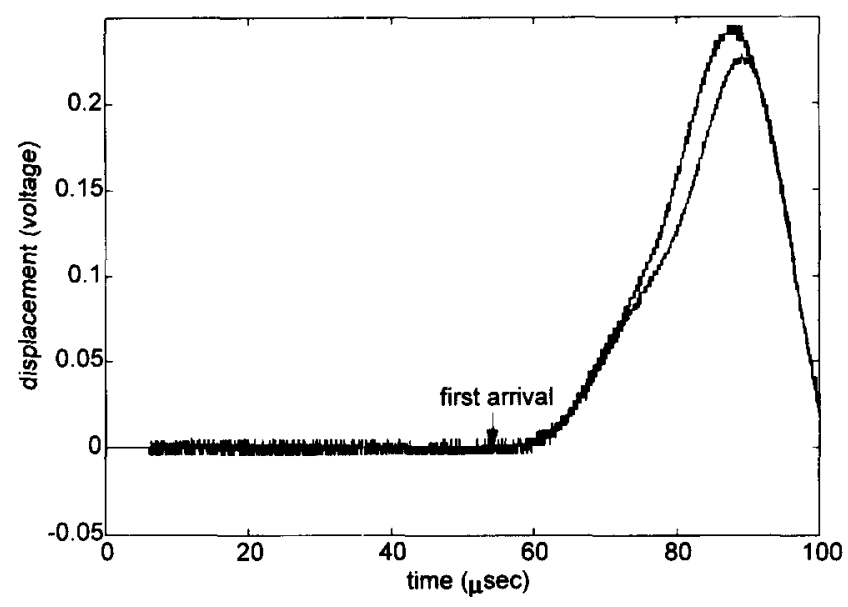

Figure 11 Displacement curve of the vertical model crack

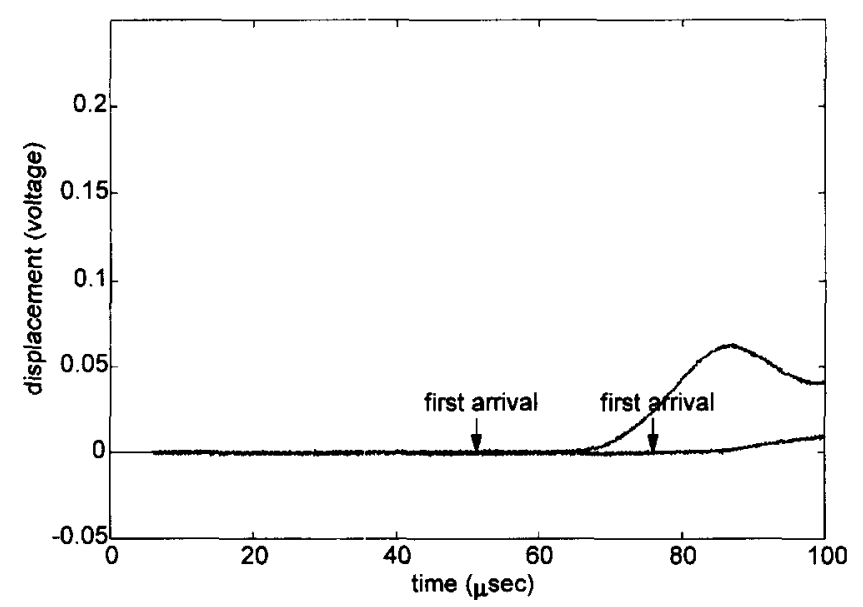

Figure 12 Displacement curve of the $45^{\circ}$ model crack

$100 \mathrm{MHz}$ digital oscilloscope (LeCroy 9314L).

Two experiments were conducted on each specimen. In the first experiment, $s_{1}=-10 \mathrm{~mm}$ and $r_{1}=100 \mathrm{~mm}$; in the second experiment, $s_{2}=10 \mathrm{~mm}$ and $r_{2}=-100 \mathrm{~mm}$.

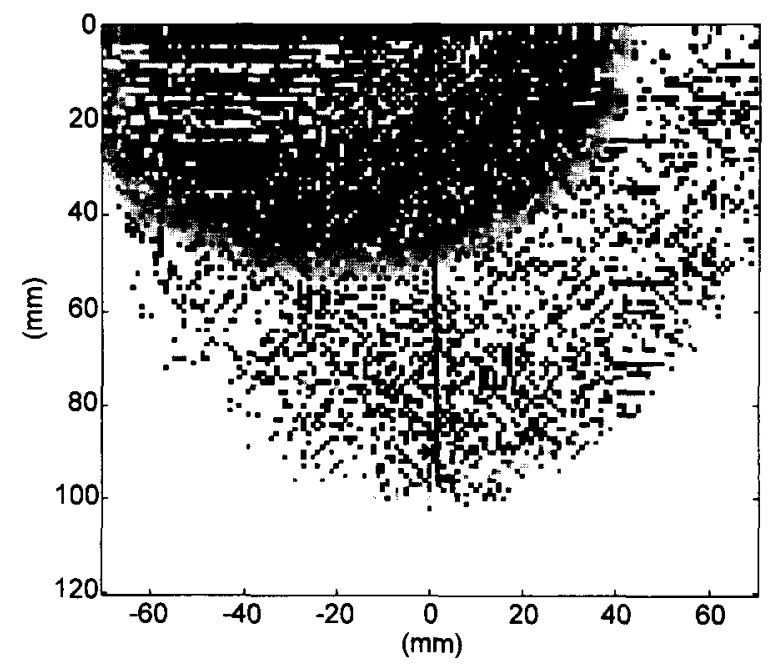

Figure 13 Image of the vertical model crack using the original displacement curve

Figures 11 and 12 show the vertical displacements on the surfaces of the two specimens.

First, consider the vertical crack. The displacement image obtained by processing the original time traces is shown in Figure 13. In the interpolation process, the interpolation length is $D=4 \mu \mathrm{s}$ and the threshold is $T=4.5 \times 10^{-3} \mathrm{~mm}$.

The zero-greyscale zone is still observable in the displacement image. Although the zone is speckled due to the presence of noise, its V-shaped corner still can be found. However, the zero-greyscale zone image is not symmetric with respect to the vertical crack. This is because the two traces are not identical.

The displacement traces can be window-averaged or filtered to attain a better image of the crack. Applying a 200 -point window averaging to the traces, one obtains the clean displacement image in Figure 14. The length of the crack obtained by quadratic interpolation is $96.7 \mathrm{~mm}$, $7.5 \%$ longer than the actual crack length, and the dip angle is $89.1^{\circ}$.

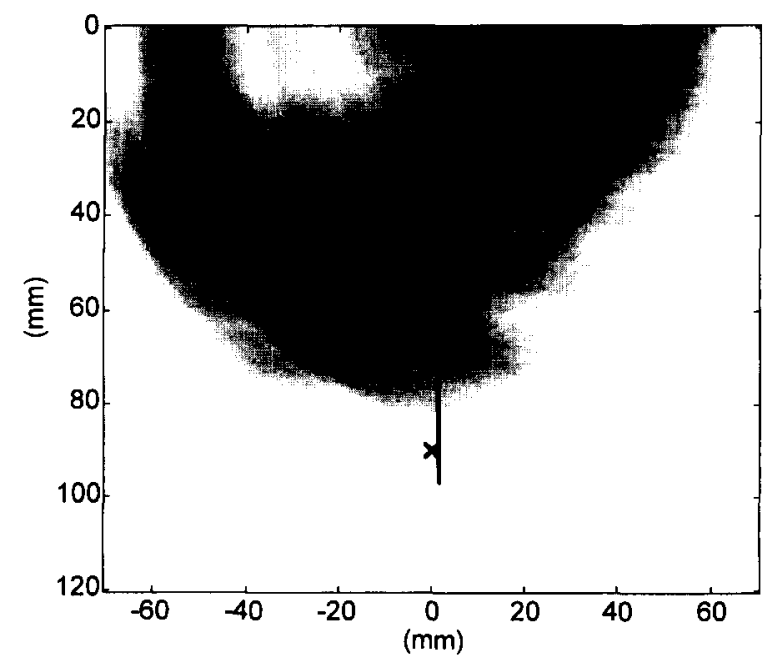

Figure 14 Image of the vertical model crack using the windowaveraged displacement curve 


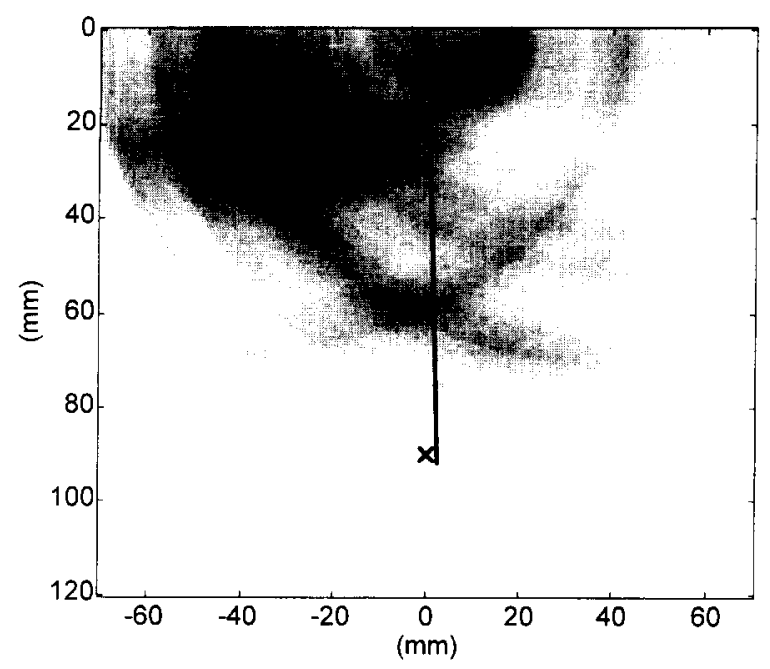

Figure 15 Image of the vertical model crack using the windowaveraged velocity curve

It is shown in the numerical examples that the velocity images depict the crack tip better than the displacement image. Theoretically, the velocity curve can be obtained from the original displacement curve by finite difference. However, the experimental signals contain noise. Therefore, the velocity curves are obtained by differentiating the window-averaged displacement curves. Windowaveraging is performed again on the velocity curves before imaging.

The velocity image of the vertical crack is shown in Figure 15. Similar to the numerical example, the velocity image is better than the displacement image. The length of the crack obtained by quadratic interpolation is $91.8 \mathrm{~mm}$, which is $2 \%$ longer than the actual crack length. The dip angle is $88.4^{\circ}$.

Next, consider the $45^{\circ}$ crack. The displacement and velocity images of the $45^{\circ}$ crack are shown in Figure 16 . Both images are constructed from window-averaged traces. The crack tip can be pin-pointed in the two images. The velocity image is again superior to the displacement image. The crack tips obtained by quadratic interpolation are very close to the actual tip location in both images.

These model tests verify that the proposed imaging method can display real cracks successfully.

\section{Conclusions}

This paper presents a method to construct the image of a surface-breaking crack. The imaging method includes two steps: first, the migration method in reflection seismology is adopted to construct the image of the crack tip. Second, the quadratic interpolation is applied to determine the location of the crack tip. Then, a solid line is superposed on the image to represent the crack.

Three numerical examples and two model tests are presented to illustrate the effectiveness of the proposed

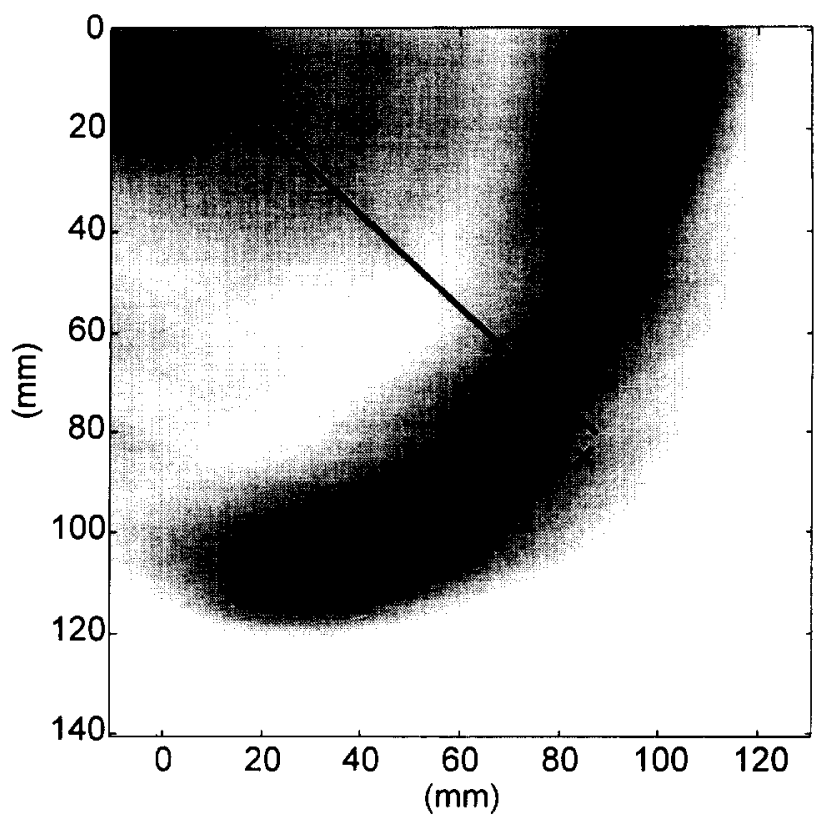

(a)

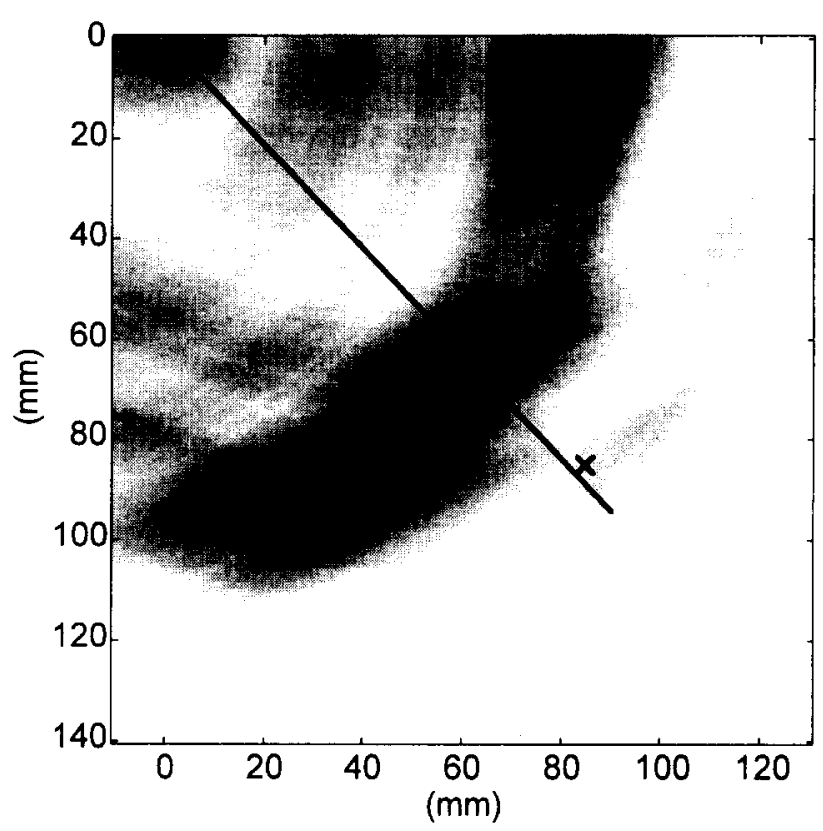

(b)

Figure 16 (a) Displacement and (b) velocity images of the $45^{\circ}$ model crack

method. Several conclusions can be drawn from this study:

1. In order to avoid the diffracted wave being overwhelmed by the strong surface wave, the source and receiver must be placed on the opposite sides of the crack opening.

2. In order to make the crack tip clearly visible in the image, the layout of the source and receiver should be carefully designed. 
3. The velocity image is superior to the displacement image.

4. The proposed method can display the crack image successfully.

The proposed method provides the inspector with most direct information on the concrete interior. Therefore, the image method can serve as a useful tool in the nondestructive evaluation of a concrete structure.

\section{Acknowledgement}

This work was supported by the National Science Council, Taiwan, Republic of China under Grant NSC 84-2621-P-002-011B.

\section{References}

1 Lin, $\mathbf{Y}$. and Sansalone, $M$. 'Detecting flaws in concrete beams and columns using the impact-echo method' Mater J Amer Concr Inst July/August (1992) pp 394-405

2 Wu, T.-T., Fan, J.-S., Liu, G.-Y., and Kuo, M.-K. 'Determination of elastic constants of a concrete specimen using transient elastic waves' J Acoust Soc Amer 984 (1995) pp 2142-2148

3 Wu, T.-T. and Fang, J.-S. 'Crack depth determination of a concrete specimen using phase identification of transient elastic waves' J Clin Inst Civ Hydraul Eng 83 (1996) pp 337-342

4 Lin, Y. and Su, W. C. 'The use of stress waves for determining the depth of surface-opening cracks in concrete structures,' Mater $J$ Amer Concr Inst to appear

5 Waters, K. H. Reflection Seismology John Wiley, New York (1981)

6 Virieux, J. (1986) 'P-SV wave propagation in heterogeneous media: velocity-stress finite-difference method' Geophys 51 (1986) pp 889-901

7 Wu, T.-T. and Gong, J.-H. 'Application of transient elastic waves to the nondestructive evaluation of plate structure with cavity or inclusion' J Acoust Soc Amer 943 (1993) 1453-1460 